\title{
Special feature on new directions in econophysics
}

\author{
Wataru Souma ${ }^{1}$ Yoshi Fujiwara ${ }^{2}$
}

Published online: 7 November 2016

(C) Japan Association for Evolutionary Economics 2016

H. Eugene Stanley proposed the term "Econophysics" in 1995 when he organized the conference of statistical physics held in Kolkata, India. The inaugural meeting of econophysics was held in Budapest in 1998. After this conference, annual meetings of econophysics have been held, including the Workshop on Economic Science with Heterogeneous Interacting Agents (WEHIA, since 1995), Applications of Physics in Financial Analysis (APFA, 1999-2009), Econophysics Colloquium (since 2005), and ECONOPHYS-KOLKATA (since 2005).

In Japan, there are two major domestic workshops on econophysics. One was started by Hideaki Aoyama and has been held every other year since 2003 at the Yukawa Institute for Theoretical Physics (here referred to as the YITP econophysics workshop). The other was started by Mieko Tanaka and has been held almost twice annually since 1998 at the Institute of Statistical Mathematics (ISM).

The YITP econophysics workshop aims to develop econophysics together with physicists, economists, mathematicians, sociologists, information scientists, and businesspersons. In addition, the YITP econophysics workshop focuses on the establishment of "macro-econophysics," a term proposed by Hiroshi Yoshikawa.

The Japanese econophysics community has been investigating business and financial networks from 2000, prior to the global trend of studying this subject, and the YITP econophysics workshop has played a significant role in the advancement of this field. The results of YITP econophysics workshop have been summarized in Aoyama et al. (2009, 2012).

This special feature is the fruit of the YITP econophysics workshop 2015, held on December 3 and 4, 2015, and contains many topics, for example, financial analysis, financial modeling, firm size, network science, text mining, etc. The recent trend in

\footnotetext{
Wataru Souma

souma.wataru@nihon-u.ac.jp

1 Nihon University, Tokyo, Japan

2 University of Hyogo, Kobe, Japan
} 
econophysics is further fusion with diverse fields such as information science and computer science.

The subtitle of this workshop was "seeking for the new direction." In the special session of the workshop, we discussed what econophysics should be in the future, which resulted in two problems to solve. One is the application of econophysics to economic policy, and the other is the prediction of financial crises. It is expected that both problems will be solved by real-time simulation using supercomputers and big data. Hence, econophysics and the next-generation computer simulations using the "Post-K" computer will be hot topics in the forthcoming YITP econophysics workshop 2017.

\section{References}

Aoyama H, Fujiwara Y, Iyetomi H, Sato AH (eds) (2009) Econophysics: physical approach to social and economic phenomena. Proceedings of the YITP workshop on econophysics 2007. Progress of theoretical physics supplement, vol. 179

Aoyama H, Fujiwara Y, Iyetomi H, Sato AH (eds) (2012) Econophysics 2011: the Hitchhiker's Guide to the economy. Proceedings of the YITP workshop on econophysics. Progress of theoretical physics supplement, vol. 194 